\title{
Continuum Theory of Nanostructure Decay Via a Microscale Condition
}

\section{Citation}

Margetis, Dionisio, Pak-Wing Fok, Michael J. Aziz, and Howard A. Stone. 2006. Continuum theory of nanostructure decay via a microscale condition. Physical Review Letters 97(9): $096101-096104$.

\section{Published Version}

http://dx.doi.org/10.1103/PhysRevLett.97.096102

\section{Permanent link}

http://nrs.harvard.edu/urn-3:HUL.InstRepos:2794937

\section{Terms of Use}

This article was downloaded from Harvard University's DASH repository, and is made available under the terms and conditions applicable to Other Posted Material, as set forth at http:// nrs.harvard.edu/urn-3:HUL.InstRepos:dash.current.terms-of-use\#LAA

\section{Share Your Story}

The Harvard community has made this article openly available.

Please share how this access benefits you. Submit a story.

Accessibility 


\title{
Continuum Theory of Nanostructure Decay Via a Microscale Condition
}

\author{
Dionisios Margetis, ${ }^{1, *}$ Pak-Wing Fok, ${ }^{1}$ Michael J. Aziz, ${ }^{2}$ and Howard A. Stone ${ }^{2}$ \\ ${ }^{1}$ Department of Mathematics, Massachusetts Institute of Technology, Cambridge, Massachusetts 02139, USA \\ ${ }^{2}$ Division of Engineering and Applied Sciences, Harvard University, Cambridge, Massachusetts 02138, USA
}

(Received 29 June 2006; published 1 September 2006)

\begin{abstract}
The morphological relaxation of faceted crystal surfaces is studied via a continuum approach. Our formulation includes (i) an evolution equation for the surface slope that describes step line tension, $g_{1}$, and step repulsion energy, $g_{3}$; and (ii) a condition at the facet edge (a free boundary) that accounts for discrete effects via the collapse times, $t_{n}$, of top steps. For initial cones and $t_{n} \approx \tilde{t} n^{4}$, we use $\tilde{t}(g)$ from step simulations and predict self-similar slopes in agreement with simulations for any $g=g_{3} / g_{1}>0$. We show that for $g \gg 1$, (i) the theory simplifies to an equilibrium-thermodynamics model; (ii) the slope profiles reduce to a universal curve; and (iii) the facet radius scales as $g^{-3 / 4}$.
\end{abstract}

DOI: 10.1103/PhysRevLett.97.096102

Below the roughening temperature $T_{R}$, various structures are created on crystal surfaces, including mounds, islands, ripples, nanowires, and quantum dots [1]. Often, nominally flat crystal surfaces are covered by mounds from homoepitaxy or heteroepitaxy of semiconductors [2], metals [3], and ceramics [4], or from lithography [5]. Entire surface profiles as well as nanoscale defects such as steps are monitored [1,3] during the evolution process. The effect of such defects, i.e. nanoscale discreteness, on macroscopic surface properties and the growth and decay of nanostructures evade a complete theoretical description $[1,6]$. The incorporation of nanoscale, discrete effects into boundary conditions for a continuum description relevant to crystal decay is the subject of this Letter.

Below $T_{R}$, crystal surfaces evolve via the motion of steps [7]. Mass transport is mediated by the diffusion of point defects ("adatoms") across terraces and the attachmentdetachment of atoms at steps. There are two common theoretical approaches to crystal surface evolution. One approach [8,9] follows individual steps, incorporates widely accepted thermodynamics and kinetics, and provides details for surface morphologies, but involves too many variables to enable simple or wide-ranging predictions. Another approach [10-13] uses continuum equations for the surface height, which are easier to implement because they involve fewer variables, but is questionable $[14,15]$ near macroscopic, flat surface regions ("facets"). The relation of these two approaches is the subject of active research [6]. However, comparisons of continuum solutions with simulations of step motion have been previously limited to scaling of slope profiles with time [8] and physical parameters such as the step interaction energy [13].

In this Letter, we describe a continuum theory that incorporates the motion of top steps in the boundary conditions and predicts entire profiles in agreement with step simulations, thus consummating previous studies $[8,11,13]$. It has been recognized $[8,14,15]$ that the collapses of top steps drive macroscopic surface evolution. The inclusion in a continuum theory of a "discrete" condition,
PACS numbers: 68.35.Md, 61.46.Hk, 61.50.Ah

which replaces a boundary condition and is informed by step simulation data, is the principal contribution of our work. We treat facet evolution as a free-boundary problem via a partial differential equation (PDE) for the surface height. We show that for strong step repulsions, this theory reduces to a previous model [11,12] of equilibrium thermodynamics.

The geometry consists of a faceted axisymmetric shape (see Fig. 1). Steps of height $a$ are illustrated, which are concentric circles with radii $r_{i}(t), r_{i+1}>r_{i}, r_{0} \equiv 0$. The discrete surface height is

$$
h^{\mathrm{d}}(r, t)=h_{\text {top }}(t)-i a, \quad r_{i}<r<r_{i+1},
$$

where $h_{\text {top }}(t)$ is the (piecewise constant with time) height of the top terrace, $i$ is the step number, $i=0,1, \ldots, N, N(t)$ is the number of steps $(N \gg 1), r$ is the polar distance, and $t$ is time. We take the high-symmetry ("basal") plane $(x, y)$ of the crystal as our reference plane; $h^{\mathrm{d}}=0$ for $r>r_{N}$ and, thus, $h_{\text {top }}=N a$.

We exclude material deposition from above so that the structure relaxes to become flat. During relaxation, the top terrace (region $0<r<r_{1}$ ) shrinks mainly by the influence of the line tension of the first step. If the $n$ th-step collapse occurs at time $t_{n}, n=1, \ldots, N_{0}$, then

$$
\dot{N}(t)=-\sum_{n} \delta\left(t-t_{n}\right), \quad N(0)=N_{0} \quad\left(N_{0} \gg 1\right),
$$

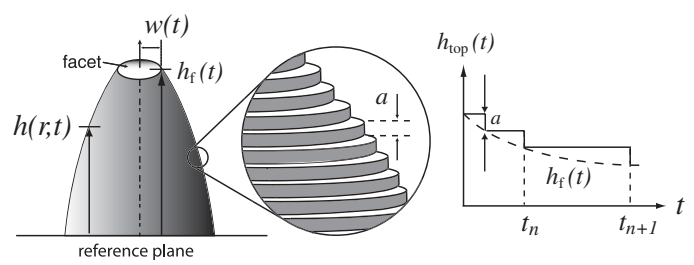

FIG. 1. Left: Schematic of an axisymmetric shape with a facet at the macroscale and nanoscale (blowup). Right: Schematic graph of piecewise constant top-terrace height, $h_{\text {top }}(t)$ (solid line), and facet height, $h_{\mathrm{f}}(t)$ (dashed line), the continuous interpolant of $h_{\text {top }}(t)$. 
where the dot over a symbol denotes the total time derivative, $\delta(t)$ is the delta function, and step collapses are counted from $t=0$. By integration of Eq. (2) from $t=$ $t_{n}^{+}$(i.e., $t \approx t_{n}$ with $t_{n}<t<t_{n+1}$ ) to $t_{n+1}^{+}$, we obtain

$$
N\left(t_{n+1}^{+}\right)-N\left(t_{n}^{+}\right)=-1, \quad n=1,2, \ldots, N_{0},
$$

which is a special case of Eq. (2).

Next, we reduce Eq. (3) to a continuum statement. The step density varies over a macroscopic length $\lambda(a / \lambda \ll$ $1)$. Then, $h^{\mathrm{d}} \approx h(r, t)$, which is the continuous height profile; $h(r, t)=h_{\mathrm{f}}(t)$ for $r<w(t)$, where $h_{\mathrm{f}}$ is the timecontinuous facet height and $w$ is the facet radius (see Fig. 1). Because $h(r, t)$ is an interpolation of the discrete height, $h^{\mathrm{d}}$, then $h_{\mathrm{f}}=h_{\text {top }}+O(a)$ so that $h_{\mathrm{f}}(t) \approx h_{\text {top }}(t)$ in the limit $a / \lambda \rightarrow 0$; in a similar way, $t \approx t_{n}$. Thus, Eq. (3) with $h_{\text {top }}(t)=N(t) a$ yields

$$
h_{\mathrm{f}}\left(t_{n+1}\right)-h_{\mathrm{f}}\left(t_{n}\right)=-a,
$$

which is viewed as a discrete scheme for $h_{\mathrm{f}}(t)$; we call this relation the "step-drop" condition.

Equation (4) was introduced but not implemented by Israeli and Kandel [8] [see their Eq. (42)]. Extensions of this relation are discussed in [16]. A fully continuum statement is obtained by replacing Eq. (4) by

$$
\Delta t \dot{h}_{\mathrm{f}}(t)=-a,
$$

where the meaning of $\Delta t$ is discussed next. By taking $\Delta t \approx$ $t_{n+1}-t_{n}$ and $t_{n}=T(n)$, we have $\Delta t(t) \approx T^{\prime}(n)$, where the prime denotes differentiation. The discrete function $T(n)$ depends on the initial step positions. Equation (5) relates the times of step collapses $\left(t_{n}\right)$ with the continuous height function $h_{f}$. Similar, hybrid-type conditions have been used elsewhere in continuum solid mechanics [17].

To compute $t_{n}$ and the discrete step density or surface slope, $m_{i}(t)=\frac{a}{r_{i+1}-r_{i}}$, for later comparisons with continuum predictions, we next review and solve numerically the equations of step motion $[8,13]$. The $i$ th-step velocity is $\dot{r}_{i}=\frac{\Omega}{a}\left(J_{i-1}-J_{i}\right)$ at $r=r_{i}(t)$, where $\Omega$ is the atomic volume, $J_{i}(r, t)=-D_{\mathrm{s}} \frac{A_{i}}{r}$ is the adatom current on the $i$ th terrace $\left(r_{i}<r<r_{i+1}\right)$, and $D_{\mathrm{s}}$ is the terrace diffusivity; the coefficient $A_{i}$ is proportional to the difference $\mu_{i+1}-\mu_{i}$ [13], where

$$
\mu_{i}=\frac{\Omega g_{1}}{r_{i}}+\frac{\Omega}{2 \pi a r_{i}} \frac{\partial\left[V\left(r_{i}, r_{i+1}\right)+V\left(r_{i-1}, r_{i}\right)\right]}{\partial r_{i}}
$$

is the chemical potential of the $i$ th-step. Here, $g_{1}$ is the step line tension, and $V\left(r_{i}, r_{i+1}\right)=g_{3} \frac{4 \pi a^{3} r_{i} r_{i+1}}{\left(r_{i+1}+r_{i}\right)\left(r_{i+1}-r_{i}\right)^{2}}$ is the step interaction energy; it is convenient to characterize $\mu_{i}$ in terms of the parameter $g=g_{3} / g_{1}$. This formulation yields coupled ordinary differential equations (ODEs) for $r_{i}(t)$, which we solve for diffusion-limited (DL) kinetics (when terrace diffusion is the rate-limiting process) and an initial linear cone of unit slope.

To capture the dynamics of a structure for which the base does not influence the facet motion, we use a sufficiently large number of steps and test that the discrete step density approaches unity with high accuracy away from the facet. We verify that the step density becomes self-similar [8], i.e. $m_{i} \equiv M\left(\xi_{i}\right)$, where $\xi_{i} \propto r t^{-1 / 4}$ and $r=\frac{r_{i}+r_{i+1}}{2}$; details are given in [16]. We find numerically that $t_{n} \approx \tilde{t}^{4}$, a scaling that was also reported in [8]. Below, we predict this scaling by applying continuum ideas, compute $\tilde{t}$ by step simulations, and use it as an input to the continuum theory via Eq. (5). In Fig. 2, we plot the scaled $\tilde{t}$ as a function of $g$; we note its weak dependence on small values of $g$. Further, from the ODE for the top step we find $\tilde{t}=O\left(g^{-1}\right)$ for $g \gg$ 1 and fixed $g_{1}$. In the following, we show the importance of $\tilde{t}$ for linking the discrete step simulations with a continuum description.

So far, emphasis is on the discrete step motion, which enters Eq. (5) via $\Delta t(t)$. We now outline the continuum principles that yield an evolution equation for the surface height, $h$; a detailed derivation is given in [13]. Our starting point is the surface free energy per projected area, $G(m)=$ $g_{0}+g_{1} m+\frac{1}{3} g_{3} m^{3}$, where $m=|\nabla h|, g_{0}$ is the energy of a flat surface and $\boldsymbol{\nabla}=\left(\frac{\partial}{\partial x}, \frac{\partial}{\partial y}\right)$. The continuum step chemical potential, $\mu$, is the continuum limit of Eq. (6), or the variational derivative of the total surface free energy [13]; and the continuum surface current for DL kinetics is $\mathbf{J} \propto-\nabla \mu$. A PDE for the surface slope, $m(r, t)=$ $-\frac{\partial h}{\partial r}(m>0)$, outside the facet, $r>w(t)$, is obtained via mass conservation, $\frac{\partial h}{\partial t}+\Omega \boldsymbol{\nabla} \cdot \mathbf{J}=0$ :

$$
\frac{1}{B} \frac{\partial m}{\partial t}=\frac{3}{r^{4}}-g \frac{\partial}{\partial r} \frac{1}{r} \frac{\partial}{\partial r} r \frac{\partial}{\partial r} \frac{1}{r} \frac{\partial}{\partial r}\left(r m^{2}\right), \quad r>w,
$$

where $B$ is a material parameter $(B>0)$ and each space derivative acts on the entire term on its right.

Next, we solve Eq. (7) by treating the facet edge, $r=$ $w(t)$, as a free boundary [11]. We consider an initial cone and self-similar slope profiles, $m(r, t) \equiv m(\xi ; g)$, where $\xi=(B t)^{-1 / 4} r$. Then, Eq. (7) reduces to the ODE

$$
-\frac{\xi}{4} \frac{d m}{d \xi}=\frac{3}{\xi^{4}}-g \frac{d}{d \xi} \frac{1}{\xi} \frac{d}{d \xi} \xi \frac{d}{d \xi} \frac{1}{\xi} \frac{d}{d \xi}\left(\xi m^{2}\right), \quad \xi>\xi_{0},
$$

where $\xi_{0}=(B t)^{-1 / 4} w$ and $w=O\left((B t)^{1 / 4}\right)[8,13]$.

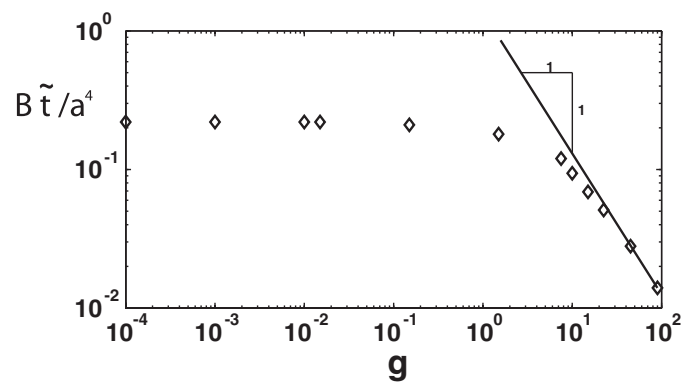

FIG. 2. The scaled step collapse time $\tilde{t}, \tilde{t}=t_{n} / n^{4}$ for $n \gg 1$, as a function of the relative step interaction strength, $g$, for an initial cone of unit slope; $B$ is a material parameter. Symbols are step simulation data and the solid line is our prediction, $\tilde{t}=$ $O\left(g^{-1}\right)$ for $g \gg 1$. 
We solve Eq. (8) by applying two distinct sets of boundary conditions. One set follows from equilibrium thermodynamics [11-13] and includes continuity of slope, height, current, and a continuous extension of the step chemical potential (" $\mu$-continuity") across the facet edge. The first condition amounts to imposing $m=0$ at $\xi=\xi_{0}$, to be justified later. Continuity of height, $h(w, t)=h_{\mathrm{f}}(t)$, gives $\frac{\partial h}{\partial t}=\dot{h}_{\mathrm{f}}$ at $r=w$; by Eq. (7), at $\xi=\xi_{0}$ we have

$$
-\kappa \xi_{0}^{3}=1+g \xi_{0}\left[-\left(m^{2}\right)_{\xi}+2 \xi_{0}\left(m^{2}\right)_{\xi \xi}+\xi_{0}^{2}\left(m^{2}\right)_{\xi \xi \xi}\right],
$$

where $\left(m^{2}\right)_{\xi} \equiv \frac{d m^{2}}{d \xi}$ and the parameter $\kappa$ is defined by

$$
\kappa \equiv-\dot{h}_{\mathrm{f}} B^{-1}(B t)^{3 / 4}>0 \text {. }
$$

Continuity of current asserts that $J_{\mathrm{f}}(w, t)=J(w, t)$ where $J \propto-\frac{\partial \mu}{\partial r}$ and $J_{\mathrm{f}}(r, t)$ is the current on the facet, which is defined in terms of $\dot{h}_{\mathrm{f}}$ via mass conservation, $\dot{h}_{\mathrm{f}}+$ $\Omega r^{-1} \frac{\partial}{\partial r}\left(r J_{\mathrm{f}}\right)=0$. Thus, we obtain [13]

$$
\kappa \xi_{0}^{3} / 2=1-g \xi_{0}\left[\left(m^{2}\right)_{\xi}+\xi_{0}\left(m^{2}\right)_{\xi \xi}\right], \quad \xi=\xi_{0} .
$$

The continuous extension $\mu_{\mathrm{f}}(r, t)$ of $\mu$ onto the facet is defined via $J_{\mathrm{f}} \propto-\frac{\partial \mu_{f}}{\partial r} ; \mu$-continuity asserts that $\mu_{\mathrm{f}}=\mu$ at $r=w$, which yields [13]

$$
\kappa \xi_{0}^{3} / 8=1-g \xi_{0}\left(m^{2}\right)_{\xi}, \quad \xi=\xi_{0} .
$$

The ODE (8) is solved uniquely with $m=0$ at $\xi_{0}$, Eqs. (9)-(12), and two more conditions by $m \rightarrow 1$ as $\xi \rightarrow$ $\infty$. Therefore, $\kappa$ and hence $\dot{h}_{\mathrm{f}}$ can be eliminated from the boundary conditions; the vertical motion of the facet described by $\dot{h}_{\mathrm{f}}$ is absent from this formulation.

By contrast, to retain the influence of the discrete topstep motion on continuum solutions, we replace Eq. (12) by the step-drop condition (5). The latter links the macroscopic facet motion via $\dot{h}_{\mathrm{f}}$ with the microscale top-step collapses in simulations via $\Delta t(t)$. Because by Eq. (9) or (11) $\kappa$ must be independent of $t$, by Eq. (10) we find $\dot{h}_{\mathrm{f}}=$ $O\left(t^{-3 / 4}\right)$. Thus, by Eq. (5), $\Delta t=O\left(t^{3 / 4}\right)=t_{n+1}-t_{n}$, which implies $t_{n}=T(n) \sim \tilde{t}(g) n^{4}$, in agreement with the results in [8]; $\tilde{t}(g)$ is determined by step simulations. Equation (5), which describes the time change in the facet height, becomes

$$
\kappa=\frac{a}{4(B \tilde{t})^{1 / 4}}
$$

in Fig. 2, we plot $(4 \kappa)^{-4}$ as a function of $g$. This condition relates $\dot{h}_{f}$ with $\tilde{t}$. We interpret this relation as a means to construct the time-continuous $h_{\mathrm{f}}(t)$ from $h_{\text {top }}(t)$ (see Fig. 1). Equation (13) was first proposed in [8] but used only to extract estimates for $\tilde{t}$ when $g<1$; to our knowledge, Eq. (13) has not been previously implemented in a continuum setting. For $\tilde{t}$, or $\kappa$, given by step simulations, Eq. (8) is solved uniquely with $m=0$ at $\xi_{0}$, conditions (9), (11), and (13), along with $m \rightarrow 1$ as $\xi \rightarrow \infty$.
We now discuss features of the continuum solutions with particular emphasis on results obtained by the step-drop condition. In Figs. 3(a) and 3(b), we compare results of step simulations to continuum predictions obtained by (8) with the two sets of boundary conditions. We make the following observations. First, the slope, $m$, extrapolated from the step simulation data rapidly approaches zero for decreasing $\xi$ and any $g>0$; this behavior confirms the condition $m=0$ at $\xi_{0}$. Second, the $\mu$-continuity generates slope profiles that deviate significantly from the step simulation results when $g<1$. By contrast, slope profiles from the step-drop condition follow closely the discrete simulation data for arbitrary $g>0$. Third, the two sets of boundary conditions produce nearly identical results for large enough $g$; in this case, the facet width decreases to zero. We note that previous ODE solutions [13] using $\mu$-continuity, which appeared to agree with step simulation results, were in error due to a fault in the numerical implementation; nevertheless, the small- $g$ scaling laws in [13] are correct. We reiterate that the continuum results in agreement with step simulations come from the step-drop condition, which incorporates the effect of the top-step motion.

Next, we explain the behavior of the two families of continuum solutions (with and without $\mu$-continuity), especially their coalescence when the step interactions be-
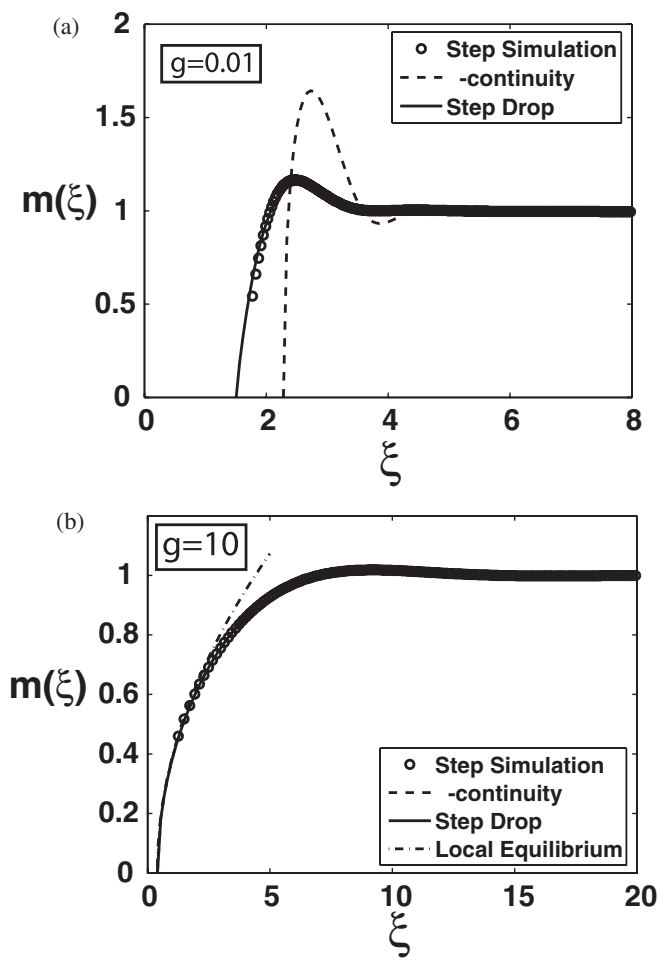

FIG. 3. Self-similar slope, $m$, for an initial cone of unit slope. Step simulation data (circles) and continuum solutions of Eq. (8) by $\mu$-continuity (dashed line) and step-drop condition (solid line) for: (a) $g=0.01$; and (b) $g=10$. The dot-dashed line is our large- $g$ prediction, $m \sim g^{-1 / 8} \sqrt{\frac{\xi-\xi_{0}}{\check{\xi}_{0}}}$. 


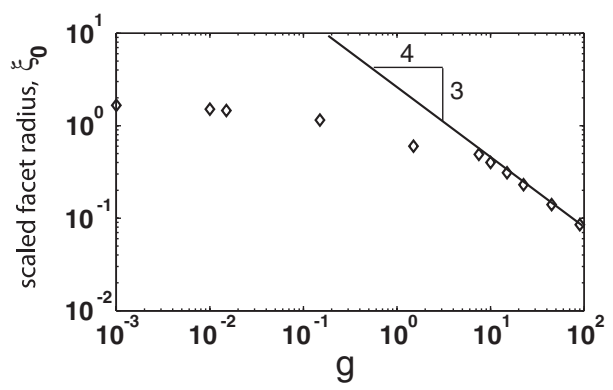

FIG. 4. The (scaled) facet radius, $\xi_{0}=w(B t)^{-1 / 4}$, as a function of $g$. Circles correspond to step simulations and the solid line is our continuum prediction, $\xi_{0}=O\left(g^{-3 / 4}\right)$ for $g \gg 1$.

come dominant, $g \gg 1$. In the governing ODE, Eq. (8), we balance the step interaction ( $g$-) term with $\xi \frac{d m}{d \xi}$; the latter comes from the time derivative, $\frac{\partial m}{\partial t}$, in Eq. (7), and is necessary for satisfying the far-field condition. Thus, we define the variable $\chi=g^{-1 / 4} \xi$ so that $m(\xi ; g) \equiv \tilde{m}(\chi)$; by treating $\tilde{m}$ and its derivatives with respect to $\chi$ as bounded, we simplify Eq. (8) for $g \gg 1$ to the $g$-independent equation

$$
\frac{d}{d \chi} \frac{1}{\chi} \frac{d}{d \chi} \chi \frac{d}{d \chi} \frac{1}{\chi} \frac{d}{d \chi}\left(\chi \tilde{m}^{2}\right)=\frac{\chi}{4} \frac{d \tilde{m}}{d \chi}, \quad \chi>\chi_{0}
$$

$\chi_{0}=g^{-1 / 4} \xi_{0}$ and the term $g^{-1} \frac{3}{\chi^{4}}$ is negligible for $\chi>\chi_{0}$.

We now show that both sets of boundary conditions for Eq. (8) simplify to the same set of $g$-independent conditions for Eq. (14). By continuity of slope, we have $\tilde{m}=0$ at $\chi=\chi_{0}$. Equation (9) for height continuity becomes

$$
1-\breve{\xi}_{0}\left(\tilde{m}^{2}\right)_{\chi}=0 \quad \chi=\chi_{0} ; \quad \breve{\xi}_{0}=g^{3 / 4} \xi_{0},
$$

where neglected terms are $O\left(g^{-1 / 2}\right)$. By Eq. (15), $\breve{\xi}_{0}=$ $O(1)$ and, thus, $\xi_{0}=O\left(g^{-3 / 4}\right)$ for $g \gg 1$; this scaling behavior is compared with simulation results in Fig. 4, where $\xi_{0}(g)$ is plotted against $g$. It follows that $\chi_{0}=$ $O\left(g^{-1}\right)$; hence, to leading order in $g^{-1}$, we take $\chi_{0}=0$, by which the facet shrinks to a single point. Effectively, the surface profile is smooth (almost) everywhere and the two sets of conditions, with or without $\mu$-continuity, should become equivalent. Indeed, Eqs. (11) and (12) reduce to (15). Further, the inclusion of the step-drop condition, Eq. (13), in Eqs. (9) and (11) also yields Eq. (15) in view of $\tilde{t}=O\left(g^{-1}\right)$ for $g \gg 1$. The far-field condition remains the same, $\tilde{m} \rightarrow 1$ as $\chi \rightarrow \infty$.

Condition (15) balances two energetic contributions: (i) the step line tension, which is proportional to $1 / \breve{\xi}_{0}$ and corresponds to the energy of the top step; and (ii) the interaction energy per atom of adjacent steps, which is proportional to $\left(\mathrm{m}^{2}\right)_{\chi}$, the limit value of the continuum step chemical potential, $\mu$, at the facet edge (as $\chi \rightarrow \chi_{0}$ ).

Next, we discuss further implications of our large- $g$ analysis. The governing Eq. (14) has four, instead of the previous six, conditions: $\tilde{m}=0$ at $\chi=0$ and Eq. (15), where $\breve{\xi}_{0}$ is unknown, and the far-field behavior which amounts to two conditions [13]. By enforcing $\tilde{m}=0$, we obtain $\tilde{m}=c_{1} \chi^{1 / 2}+c_{2} \chi^{5 / 2}+O\left(\chi^{3}\right)$ as $\chi \rightarrow 0$, where only $c_{1}$ and $c_{2}$ are free. So, the problem is well posed and produces a universal, $g$-independent profile. Specifically, by Eq. (15), $c_{1}=\left(\breve{\xi}_{0}\right)^{-1 / 2}$; thus, $m(\xi ; g) \sim$ $g^{-1 / 8} \sqrt{\frac{\xi-\xi_{0}}{\breve{\xi}_{0}}}$, where $\xi_{0}<\xi<O\left(g^{1 / 4}\right)$ and $\breve{\xi}_{0}=O(1)$; corrections to this formula via Eq. (14) establish that $m \rightarrow 1$ as $\xi \rightarrow \infty$. Hence, the slope has a local-equilibrium [1] behavior for a wide range of $\xi$ (see Fig. 3(b)).

There remain further research directions for exploring the influence of discreteness on the continuum description of nanostructure evolution. For example, attachmentdetachment limited kinetics can be treated; long-range step interactions can also be included. Finite structures are the subject of work in progress. A feature of our theory is the use of the step collapse parameter $\tilde{t}$ : Currently, we compute $\tilde{t}$ from simulations, but it is possible to obtain it analytically or experimentally. Our work opens the avenue to predicting macroscale surface evolution from any source of information on the top-step motion.

We thank B. Davidovich, G.H. Gilmer, R. V. Kohn, C. A. Orme, R. R. Rosales, and J. Weeks for discussions. Work at Harvard was supported by NSF-DMR-0213805.

*Present address: Department of Mathematics and Institute for Physical Science and Technology, University of Maryland, College Park, Maryland 20742, USA

[1] H.-C. Jeong and E. D. Williams, Surf. Sci. Rep. 34, 171 (1999).

[2] J.E. Van Nostrand, S. J. Chey, and D. G. Cahill, Phys. Rev. B 57, 12536 (1998).

[3] K. Thürmer et al., Phys. Rev. Lett. 87, 186102 (2001).

[4] B. W. Karr et al., Appl. Phys. Lett. 70, 1703 (1997).

[5] S. Tanaka et al., Phys. Rev. Lett. 78, 3342 (1997).

[6] J. W. Evans, P. A. Thiel, and M. C. Bartelt, Surf. Sci. Rep. 61, 1 (2006).

[7] W. K. Burton, N. Cabrera, and F. C. Frank, Phil. Trans. R. Soc. A 243, 299 (1951).

[8] N. Israeli and D. Kandel, Phys. Rev. B 60, 5946 (1999).

[9] N. Israeli and D. Kandel, Phys. Rev. B 62, 13707 (2000).

[10] A. Rettori and J. Villain, J. Phys. (France) 49, 257 (1988); M. Ozdemir and A. Zangwill, Phys. Rev. B 42, 5013 (1990).

[11] H. Spohn, J. Phys. I (France) 3, 69 (1993).

[12] V. B. Shenoy and L. B. Freund, J. Mech. Phys. Solids 50, 1817 (2002).

[13] D. Margetis, M. J. Aziz, and H. A. Stone, Phys. Rev. B 69, 041404(R) (2004); Phys. Rev. B 71, 165432 (2005).

[14] W. Selke and P. M. Duxbury, Phys. Rev. B 52, 17468 (1995).

[15] A. Chame et al., Bulg. Chem. Commun. 29, 398 (1996/ 1997);

A. Chame and J. Villain, Phys. Rev. E 63, 026104 (2001).

[16] P.-W. Fok, Ph.D. thesis, M.I.T., 2006.

[17] P. L. O'Sullivan et al., J. Appl. Phys. 92, 3487 (2002). 